\title{
Fabrication of nanostructure arrays induced by laser on Si wafers
}

\author{
Hua Zhang* \\ School of Materials Science and Engineering, Shandong University of Technology, Zibo 255049, \\ China \\ zhanghua01979@sdut.edu.cn
}

Keywords: Semiconductors; Nanostructure Arrays; Laser processing; SEM morphology Abstract. This work reports a new method of producing nanostructure arrays on monocrystal Si wafers. During the course of electrochemical anodic etching in HF solution, nanostructure arrays formed on N-type Si wafers under laser illumination $(\lambda=650 \mathrm{~nm})$. A field emitting scanning electronic microscope (FESEM) was used to study the morphology of the samples and an X-ray energy dispersion spectroscope (EDS) was employed to analyze the components of the arrays. Effects of etching time and light sources on the morphology of nanostructure arrays obtained on polished and rough surface of silicon wafer are also studied. Moreover, the growth mechanism is proposed.

\section{Introduction}

Nanomaterials have long been a hotspot in scientific and technological community since the discovery of carbon nanowires. Many methods [1-3] have been put forward to prepare nanomaterials with special characters different from the bulk ones. Porous silicon (PS) is one of the most promising materials that can be applied in sensors [4,5] and catalysts [6] for its property of good biocompatibility, high purity and low-cost preparation. However, the nanostructures of PS are mainly nano-clusters in spongy surface tissues. While arrayed nano-semiconductor materials may have higher application values such as sub-wavelength optics [7] and high-density data storage [8]. Endeavors were made to get this marvelous structure [9-11]. PS is typically prepared with electrochemical anodic etching method in HF solution. In preparation of PS on N-type Si wafers, illumination is necessary to trigger the generation of holes in the semiconductor, which is essential in the etching process. Compared to ordinary lights in illumination, Laser has the advantages of pure, high-energy and distribution controllable.

In this paper, large-area nanostructure arrays were fabricated on Si wafers by a laser-induced method for the first time. Spherical nanostructures and Si nanowires (SiNWs) are obtained in this way. Moreover, the distribution of nanostructures on the wafer can be easily controlled by laser illumination. And to the best of our knowledge, similar research has never been reported before.

\section{Experimental}

An N-type (111) monocrystal Si wafer $(0.005 \Omega \cdot \mathrm{cm}, 350 \mu \mathrm{m}$ thick, single-side polished) was electrochemically etched in a Teflon electrolytic cell (Fig. 1) and a platinum slice was used as cathode with $3 \mathrm{~V}$ voltage applied between them. The electrolyte is a mixture of $\mathrm{HF}(40 \%)$ and $\mathrm{C}_{2} \mathrm{H}_{5} \mathrm{OH}(99.7 \%)$ with a volume ratio of $1: 1$ at room temperature. The wafer was illuminated by a $650 \mathrm{~nm}$ laser $(5 \mathrm{~mW})$ through four-layer gratings. 


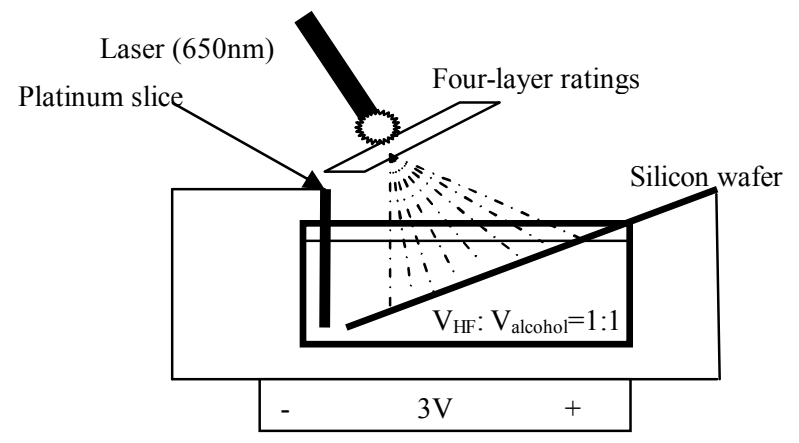

Fig. 1 Schematic of nanostructure arrays fabrication on Si wafers

Five samples are prepared in the experiment to study the influence of different factors. Preparation conditions list in Table 1 with the same electrolyte and voltage mentioned above. SEM and EDS were employed to characterize the morphology and components of obtained samples, respectively.

Table 1 Preparation conditions for different samples

\begin{tabular}{lccc}
\hline Samples & Etching time (seconds) & Wafer surface condition & Light Source \\
\hline S1 & 30 & polished & laser $(650 \mathrm{~nm}, 5 \mathrm{~mW})$ \\
S2 & 60 & polished & laser $(650 \mathrm{~nm}, 5 \mathrm{~mW})$ \\
S3 & 300 & polished & laser $(650 \mathrm{~nm}, 5 \mathrm{~mW})$ \\
S4 & 30 & polished & tungsten lamp $(20 \mathrm{~W})$ \\
S5 & 30 & rough & laser $(650 \mathrm{~nm}, 5 \mathrm{~mW})$ \\
\hline
\end{tabular}

\section{Results and Discussion}

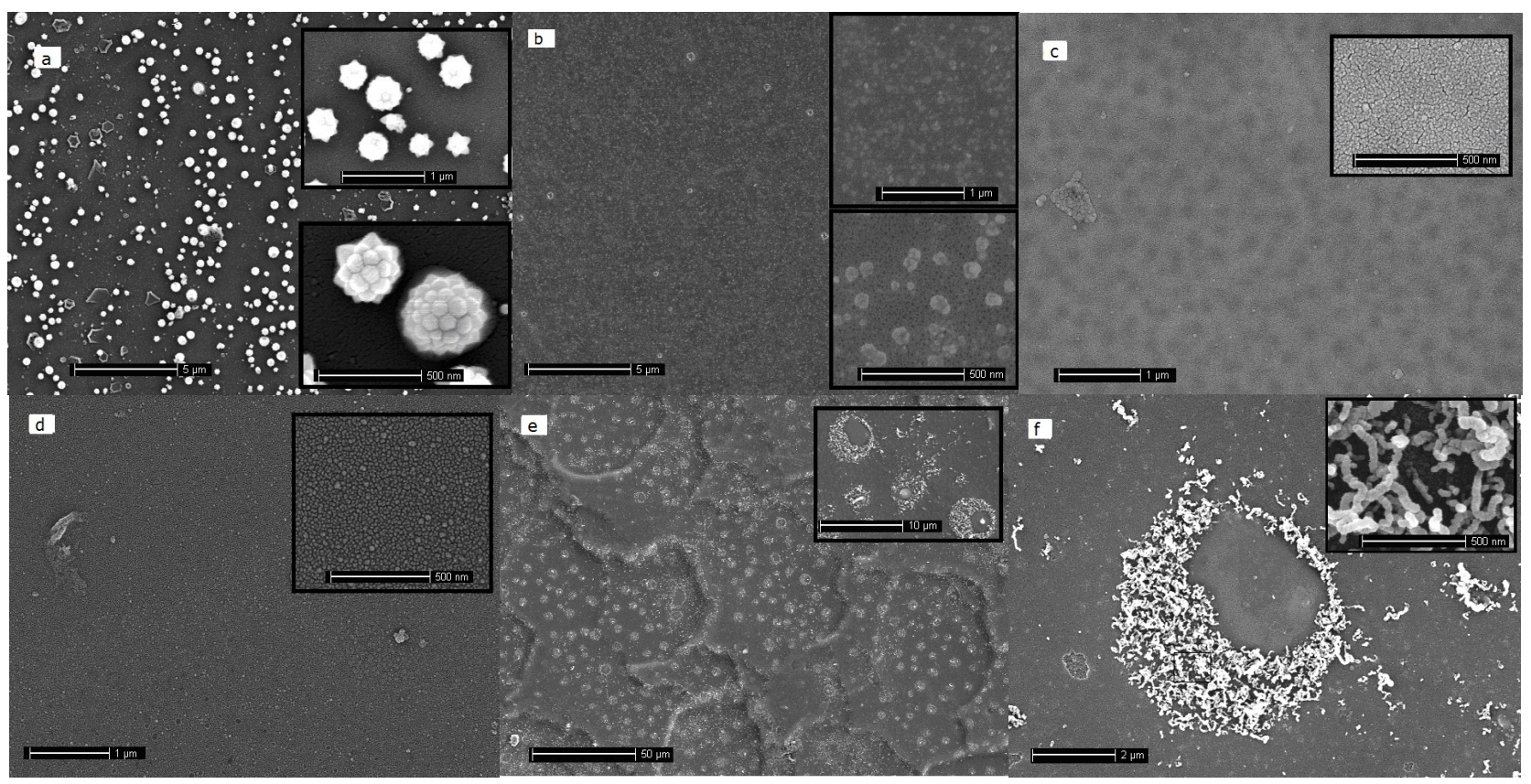

Fig. 2 SEM images of samples prepared in different conditions (a) S1 etched for 30s; (b) S2 etched for 60s; (c) S3 etched for 300s; (d) S4 etched for 30s but illuminated by a tungsten lamp; (e) (f) S5 etched for $30 \mathrm{~s}$ but etched on the rough side of the wafer

Fig.2 shows the SEM images of the samples. Fig.2a-2c shows the effects of etching time on the morphology of the samples. Spherical structures distributed in arrays on the surface of the S1 etched for 30s (Fig.2a). The diameter of the spherical structures is about $350 \mathrm{~nm}$, and further magnification shows that they are constructed by small particles of about $70 \mathrm{~nm}$ in diameter. When the etching time is prolonged to $60 \mathrm{~s}$ (S2), no obvious nanostructures but some traces were observed, as shown in Fig. $2 \mathrm{~b}$. Compared to Fig.2a, it can be inferred from the traces position and distribution that there might to be nanostructures like S1 but dissolved in the prolonged etching process. According to Fig.2c, even the 
traces like those existed in S2 disappeared when the etching time was prolonged to $5 \mathrm{~min}$. This result was in accordance with the inference before. S4 is prepared in the illumination of a tungsten lamp instead of laser, and the morphology of S4 (Fig. 2d) indicates that nanostructures can not be fabricated in the illumination of ordinary lights.

A possible mechanism is proposed to describe the formation of the nanostructure arrays. In the first stage of etching, laser spotted on the surface of the N-type Si wafer and nanostructures began to form. The laser is spotted arrayed on the wafer, so the nanostructures formed in arrays. With the growth of the nanostructures, they began to scatter laser when reached a certain size, turned unstable and began to dissolve. In the formation of nanostructure arrays, laser illumination is essential. Ordinary lights offer uniform illumination on wafers, so that the energy level of the semiconductor is uniform all over the surface, which result in no preferential etching on the wafer. As is known, uniform etching makes no arrayed structures. Besides, ordinary lights may not supply enough energy density to break the lattice structure of monocrystal to support the rapid growth of nanostructure arrays.

Effect of surface status on morphology shows in Fig.2e and Fig.2f, which were S5 etched on the rough side of the wafer for 30s. Lots of "rings" spread all over the surface of the wafer with diameter about $6 \mu \mathrm{m}$ (shown in Fig.2e) and the further magnification in Fig.2f reveals that the "rings" are constructed by huge numbers of nanowires with length more than $300 \mathrm{~nm}$. It is worth mentioning that the shapes of the rings are the same as the illumination lights of the laser. Compared to the polished sample (S1), the differences in morphology may be ascribed to the nonuniformity of the rough surface of S5. The shape of the "rings" might be caused by the insufficient focus laser that shaped in cones and casted in rings on the wafer. Compared to other ways to fabricate arrayed structures, e.g. the work by Sumio Hosaka [8] via electron beam (EB) drawing and lithography and the research made by Bahareh Yaghootkar [11], our method has the advantage of low-cost and safe.
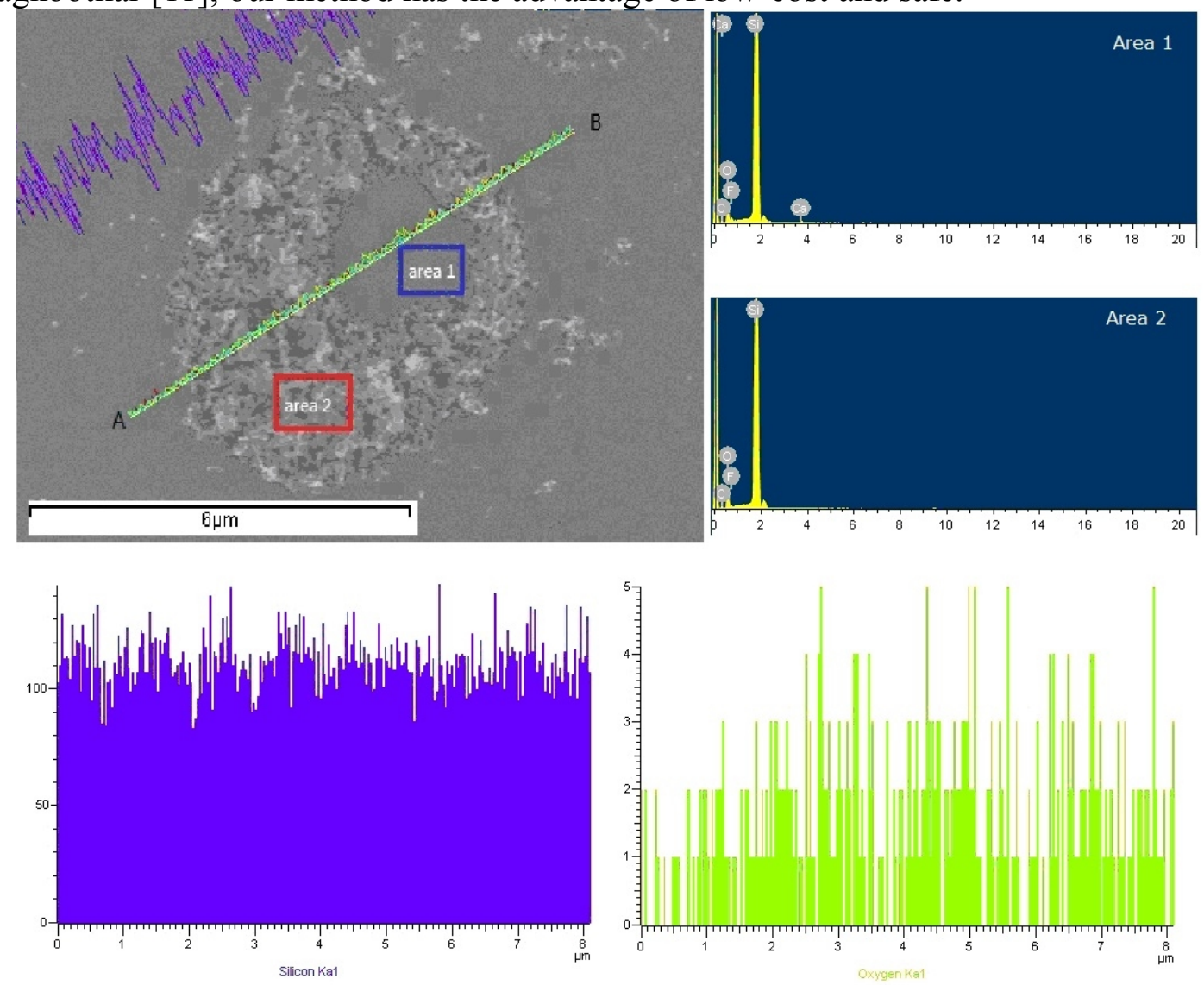

Fig. 3 EDS analysis of different areas on Si wafer (S5)

EDS was employed to analyze the elements in different areas of the wafer (Fig. 3). Two modes are employed in the EDS test, line scanning and selected area analysis. A line was selected from spot A to spot B as shown in Fig. 3 in order to distinguish different components in different surface zones on the sample. The results show that the main element is silicon, and its quantity is relatively steady in different 
zones while minute quantities of oxygen fluctuate in different zones. The oxygen content in the nanowires is about twice as those in the center of the ring and out of the ring. It may caused by oxidation of the Si nanowires that have a huge surface area.

The selected area analysis was carried out in two different areas, one in the center of the ring and the other in the nanowires. The result indicates little component differences between these two areas. A small quantity of oxygen is the result of oxidation in air. Trace amounts of fluorine and carbon come from the electrolyte and $\mathrm{CO}_{2}$ respectively. Calcium element in area1 is supposed to be a contamination in the test. According to the EDS test, the nanowires in S5 are SiNWs. Compared to other methods for SiNWs preparation[12,13], our method is simple, rapid, safe and most important of all, the distribution of the nanostructures are controllable with laser diffraction.

\section{Conclusions}

Nanostructure arrays are fabricated on Si wafers in a novel and simple laser-induced way. The nanostructures formed within $1 \mathrm{~min}$ and then dissolved as the etching prolonged. Ordinary light sources were not suitable to be used in preparation owing to their uniform illumination and low energy density. Surface conditions of the wafers only influence the morphology of the nanostructures. The EDS results confirmed that the nanowires obtained on the rough surface of the Si wafer are SiNWs. The growth mechanism of the nanostructure arrays is proposed. We believe that the intervention of laser leads preferential etching in the laser spot areas, which triggers the rapid growth of nanostructure arrays. When the nanostructures reached a certain size, they become unstable and tend to dissolve. In summary, nanostructure arrays were fabricated on the surface of N-type Si wafers in a novel and simple laser-induced way. Moreover, the shape and the position can be easily controlled by the illumination of laser, which would be very useful to corresponding applications.

\section{References}

[1]W. Li, WJ. Li, J. Wei, et al., Preparation of conductive Cu patterns by directly writing using nano-Cu ink, Mater. Chem. Phys. 146 (2014) 82-87.

[2] Neil P, Dasgupta, Shicheng $\mathrm{Xu}$, et al., Nickel Silicide Nanowire Arrays for Anti-Reflective Electrodes in Photovoltaics, Adv. Funct. Mater. 22 (2012) 3650-3657.

[3]Xiaosheng Fang, Limin $\mathrm{Wu}$, Linfeng $\mathrm{Hu}, \mathrm{ZnS}$ Nanostructure Arrays: A Developing Material Star, Adv. Mater. 23 (2011) 585-598.

[4]S. Ozdemir, J. L. Gole, A phosphine detection matrix using nanostructure modified porous silicon gas sensors, Sens. Actuators, B 151 (2010) 274-280.

[5]I. Rea, M. Iodice, G. Coppola, et al., A porous silicon-based Bragg grating waveguide sensor for chemical monitoring, Sens. Actuators, B 139 (2009) 39-43.

[6]S. Polisski, B. Goller, K. Wilson, et al., In situ synthesis and catalytic activity in CO oxidation of metal nanoparticles supported on porous nanocrystalline silicon, J. Catal. 271 (2010) 59-66.

[7]E. Højlund-Nielsen, T. Greibe, N. A. Mortensen, et al., Single-spot e-beam lithography for defining large arrays of nano-holes, Microelectron. Eng. 121 (2014) 104-107.

[8]S. Hosaka, Z. Mohamad, M. Shirai, et al., Nano-dot and -pit arrays with a pitch of $25 \mathrm{~nm} \times 25 \mathrm{~nm}$ fabricated by EB drawing, RIE and nano-imprinting for $1 \mathrm{~Tb} / \mathrm{in} 2$ storage, Microelectron. Eng. 85 (2008) 774-777.

[8]A. Narazaki, T. Sato, R. Kurosaki, et al., Nano- and microdot array formation by laser-induced dot transfer, Appl. Surf. Sci. 255 (2009) 9703-9706. 
[9]Xiaosheng Fang, Yoshio Bando, Changhui Ye, et al., Si nanowire semisphere-like ensembles as field emitters, Chem. Commun., 169 (2007) 4093-4095.

[10]Yali Li, HongYu Yu, Junshuai Li, et al., Novel Silicon Nanohemisphere-Array Solar Cells with Enhanced Performance, Small., 22 (2011) 3138-3143.

[11]B. Yaghootkar, M. Amouzgar, M. Kahrizi, A novel and low-cost multi-stage approach for the fabrication of silicon nano-structures, Sens. Actuators, A 199 (2013) 209-215.

[12]N. Bachtouli, S. Aouida, B. Bessais, Formation mechanism of porous silicon nanowires in $\mathrm{HF} / \mathrm{AgNO}_{3}$ solution, Microporous Mesoporous Mater. 187 (2014) 82-85.

[13] L. Yi, X. Bai, YM. Yang, et al., Preparation of silica nanowires using porous silicon as Si source, Appl. Surf. Sci. 258 (2011) 1470-1473. 